\title{
determinación complexométrica de trióxido de azufre en el cemento portland
}

\author{
J. CALLEJA \\ Doctor en Ciencias Químicas y Jefe del Departamento de Física y Química del I. T. C. C. \\ J. M. FERNANDEZ PARIS \\ Perito Industrial Químico de la Sección de Análisis y Ensayos del I. T, C. C.
}

Se describe un método volumétrico (complexométrico) para la determinación cuantitativa del trióxido de azufre en el cemento portland, basado en el ataque de la muestra con ácido nítrico, precipitación del trióxido de azufre con nitrato de plomo, redisolución del presipitado con tartrato sodopotásico, amortiguamiento de la disolución a $\mathrm{pH}=10 \mathrm{y}$ valoración del plomo por complexometría con EDTA (complexona III), utilizando como indicador negro de eriocromo $T$.

Los resultados concuerdan con los obtenidos por el método gravimétrico clásico (precipitación del trióxido de azufre con cloruro bárico).

La diferencia entre dos valores obtenidos por un mismo operador con la misma muestra, aplicando el método propuesto, están dentro de la tolerancia admitida por las normas A. S. T. M. para el método gravimétrico. La diferencia entre valores volumétricos y gravimétricos correspondientes a una misma muestra también entran dentro de dicha tolerancia.

El método descrito tiene la ventaja de ser más rápido, no requerir calcinación $\mathbf{y}$ poder ser aplicado por cualquier operador con los medios ordinarios de un laboratorio.

\section{Introducción}

La adición de yeso al clínker al moler este material para obtener cemento portland, es una operación indispensable, que, como es sabido, tiene una gran importancia. No sólo por el papel regulador (retardador) del fraguado que ejerce el yeso al retrasar la entrada en disolución de los aluminatos, sino por sus efectos secundarios en las resistencias mecánicas y en la retracción del cemento (1), la adición de yeso debe efectuarse con todo esmero.

Cada clínker requiere una cantidad óptima de yeso, según su composición potencial y la finura que haya de tener el cemento resultante. Esta cantidad óptima, coincidiendo, en general, con la que Lerch señala para un cemento "correctamente retardado" (2), es la que proporciona mayores resistencias mecánicas y mayor estabilidad química.

Las normas de todos los paises fijan, en vista de ello, unos valores para el contenido de trí́xido de azufre en el cemento. Suelen indicarse valores máximos, cuando, en realidad, es igualmente interesante fijar valores mínimos, $\mathrm{y}$, en todo caso, óptimos variables, dependientes de la composición potencial de cada clínker.

El antiguo Pliego español de 1930 fijaba como límite máximo de trióxido de azufre en el cemento, 2,5\%. El Pliego moderno de 1960 amplia este límite hasta $4 \%$, en vista del alto valor del contenido medio de aluminato tricálcico de los cementos españoles, y de los resultados de estualios experimentales llevados a cabo sobre el tema (3) (4). 
El trióxldo de azufre que pueda tener el clínker en si, blen sea por tener sulfatos las materias primas que se utilizan para su elaboración, o bien por aportarlo los combustibles empleados en la clinkerización, tiene también importancia, por cuanto que determina la forma en que se encuentran los álcalis en el clínker, lo que a su vez influye en la estructura y en la composición potencial de éste (5).

El control de las materias primas y combustibles para la fabricación de cemento, asi como el del producto acabado, requieren, por lo tanto, Ia determinación analítica cuantitativa de trióxido de azufre, perí́dica y frecuentemente. Como ensayo de rutina que es dentro de la fabricación de cemento, dicha determinación debe ser, en la medida de Io posible, rápida y sencilla, sin detrimento de la precisión de los resultados.

El método ordinario de determinar trlóxido de azufre en el cemento portiand consiste en la clásica preclpitación y gravimetría del sulfato bárico (6). Por otra parte, alternativamente puede efectuarse la determinación turbidimétrica utilizando el turbidimetro standard de Wagner, cuya curva de calibrado se obtiene por contraste con el procedimiento gravimétrico (7).

La gravimetria tiene el inconveniente de la lentitud de la precipitación cuantitativa del sulfato bárico y la necesidad de calcinar evitando la posible reducción del sulfato a sulfuro, mientras que la turbidimetria a nefelometría, como la colorimetria y fotometria, en general, presentan la desventaja de tener que utilizar técnicas e instrumentos especiales (8), (9), (10), (11), (12), (13) y (14).

Lo mismo sucede con los métodos espectroquímicos (11) (15), que requieren, además, una preparación especial de los operadores.

Dentro de la variedad de procedimlentos puramente químicos, que presentan alguna particularidad, cabe citar los que se basan en un intercambio ónico (16) (17) (18) (19), y los que utilizan Ia sedimentación centrifuga (20).

Por razones de rapidez y sencillez, entre los métodos químicos destacan los estrictamente volumétricos. Estos presentan a veces la diflcultad de la determinación del punto flnal de la valoración (21) (22) (23) inconveniente que se soslaya recurriendo, cuando se puede, a los procedimientos electrométricos (21) (24) (25). Aún así, éstos tienen, como los fotométricos, la desventaja de exigir un equipo especial.

Recientemente se ha ensanchado el campo de las determinaciones volumétricas con el desarrollo de la complexometría, que se ha extendido incluso al terreno del análisis industrial.

En nuestro páís se va tratando de introduclr estos métodos, y concretamente en lo que respecta al análisis del cemento portland (26).

Tiene interés para la industria del cemento la complexometria en general, tanto para el análisis de determinados componentes del cemento (27), como para el control de los crudos y materias primas que intervienen en su fabricaclón (28).

Se han desarrollado métodos para la determinación complexométrica de sulfatos en general (29) (30) (31) (32) (33), así como en el caso específico de los cementos (12) (29) (34). De ellos parecen particularmente interesantes los que se basan en la disolución del precipitado del sulfato bárico en un exceso de complexona (EDTA) y valoración del exceso de ésta (29) o los que se fundan en la adiclón de un exeeso de disolución de cloruro bárico de concentración conocida y determinación complexométrica del exceso de bario, y aquellos en que se valora directamente el precipitado, bien sea de sulfato bárico o de sulfato de plomo, utilizando distíntos indicadores.

Los primeros tienen el inconveniente, en el caso del cementa, de no evitar la precipitación, siempre Ienta, del sulfato bárico, asi como la diflultad de disolver el preclpitado una vez formado si se utiliza este recurso.

Los segundos presentan las desventajas siguientes (12): en primer lugar, los indicadores no son específicos de los iones bario o plomo, por lo que deben ser previamente elíminados otros cationes presentes en el cemento, como el calcio, el magnesto y el hierro, que interfleren; en segundo lugar, para lograr una precipitación rápida debe operarse en medio alcohólico, en el cual las sales extrañas disueltas precipitan parcialmente diflcultando ia observación del punto final; en tercer lugar, si se quiere evitar este inconveniente debe recurrirse al empleo de cambiadores de iones (35).

\section{Parte experimental}

Por todas las diflcultades señaladas, puestas de maniffesto al intentar la disolución del precipitado de sulfato bárico o al valorar por retroceso el exceso de bario, se recurrí́ a un procedimiento distinto, consistente en el ataque de la muestra de cemento con ácido nítrico, precipitación de los sulfatos con nitrato de plomo, disolución con tartrato sodo-potásico del preclpitado del sulfato de plomo formado, y valoración del plomo con EDTA, empleando como indicador negro de eriocromo $\mathbf{T}$.

Se determinó el contenido de trióxido de azufre en 10 cementos portland distintos, En cada caso se aplicó el método gravimétrico clásico (6), asi como el complexométrico que se propone, haciendo tanto por uno como por otro, determinaciones duplicadas de cada muestra. 


\subsection{Método complexometrico.}

En detalle es como sigue:

Se pesa 1 gramo de cemento, tomado de una muestra previamente desecada en estufa a $110^{\circ} \mathrm{C}$ Se pasa a un vaso de vidrio al que se añaden $25 \mathrm{ml}$ de agua destilada y $3 \mathrm{ml}$ de ácido nítrico concentrado $(d=1,43)$. No debe sobrepasarse esta cantidad. Por medio de una varllla de vidrio se deshacen los grumos que pudieran formarse. Se añaden otros $25 \mathrm{ml}$ de agua destilada y caliente y se agita. Se coloca el vaso, tapado con un vidrio de reloj, sobre un baño maria en el que se mantiene durante medla hora.

Una vez completado el ataque, se filtra a través de un fltro de papel Schleicher y Schüll 589' (banda negra), se lava unas diez veces con agua destilada caliente, procurando que el filtrado y las aguas de lavado en conjunto no sobrepasen $\operatorname{los} 100 \mathrm{ml}$. Se recogen los líquidos en un vaso de $250 \mathrm{ml}$ de forma alta.

Se enfría el contenido del vaso hasta la temperatura ambiente lo más rápidamente posible y, una vez frio, se le añaden $10 \mathrm{ml}$ de disoluctón de nitrato de plomo al $10 \%$. La adiclón debe hacerse gota a gota y agitando, durante cinco minutos.

Se deja sedimentar el precipitado durante media hora y se flitra a través de un flitro de papel Schleicher y Scbijll 5892 (banda blanca). Se arrastra y lava el precipitado y el filtro seis veces con alcohol de $96^{\circ}$, mediante un frasco lavador.

Se pasa el flitro con el precipltado a un vaso de $500 \mathrm{ml}$ de capacidad, en el que previamente se han vertido $300 \mathrm{ml}$ de agua destilada; la concentractón del líquido no debe sobrepasar los $300 \mathrm{mg}$ por litro (34).

Se añaden $6 \mathrm{ml}$ de disolución $1 \mathrm{M}$ de tartrato sodo-potásico. A continuación, $\mathrm{y}$ gota a gota, se añade, si fuera preciso, disolución iN de hidróxido sódico hasta $\mathrm{pH}=7-8$. Se calienta a unos $50-55^{\circ} \mathrm{C}$ para que el precipitado de sulfato de plomo se disuelva totalmente.

Al líquido se afiaden $15 \mathrm{ml}$ de una disolución amortiguadora de $\mathrm{pH}=10$, formada por $54 \mathrm{~g}$ de cloruro amónico y $350 \mathrm{ml}$ de amoníaco de $25 \%$ en un litro Se adicionan también 25 gotas de la disoiución del indicador, constituída por 0,4 gramos de negro de eriocromo $T$ disueltos en $100 \mathrm{ml}$ de alcohol 1sopropilico, con la cual el líquido que se ha de valorar toma un tono de color violeta rosado. El indicador asi preparado tiene un período aproximado de utllización de un mes.

El líquido mantenido a una temperatura superior a $40^{\circ} \mathrm{C}$ se valora con una dísolución $0.05 \mathrm{M}$ de EDTA. Esta se prepara disolviendo $18,6127 \mathrm{~g}$ de la sal disódica dihidratada del ácido etilenodiaminotetracético, previamente desecada en estufa a $80^{\circ} \mathrm{C}$ durante dos horas, en agua destilada y diluyendo hasta I litro. La disolución vaiorada puede considerarse como patrón de tipo primario. En la valoración se utịizó una bureta corriente de $50 \mathrm{ml}$ de capacidad, si bien, dado el pequeño consumo de EDTA (unos $5 \mathrm{ml}$ ) pueđe utilizarse una microbureta.

El final de la valoración viene dado por el viraje del indicador a una tonalidad francamente azul, observab'e por transparencia.

Si es $\mathrm{V}$ volumen en $\mathrm{ml}$ de disolución $0,05 \mathrm{M}$ de EDTA gastado en la valoración, el tanto por ciento de trióxido de azufre en la muestra analizada vlene dado por:

$$
\mathrm{so}_{n} \%=\frac{(\mathrm{V}+0,30), 0,4}{\mathrm{P}}
$$

siendo $\mathbf{P}$ el peso exacto en gramos del cemento empleado.

En efecto, la relación estequiométrica entre el EDTA y el plomo es de mol a atomo-gramo, es decir, de $372,524 \mathrm{~g}$ del primero por cada $207,21 \mathrm{~g}$ del segundo. A 1 mol de EDTA $0,05 \mathrm{M}$, equivalente a $0,0186 \mathrm{~g}$, corresponden, por lo tanto, $0,01036 \mathrm{~g}$ de plomo, equivalentes a su vez a $0,004 \mathrm{~g}$ de trióxldo de azufre.

Por otra parte, dada la solubilidad del sulfato de plomo, $100 \mathrm{ml}$ de agua disuelven a la temperatura ordinaria $0,0045 \mathrm{~g}$ de dicha sal (33). Admitiendo que en las condiciones del análisis la solubilidad del precipitado es, aproximadamente, la misma, los $100 \mathrm{ml}$ de líquido con los que se opera retendrán $0,0045 \mathrm{~g}$ de sulfato de plomo, equivalentes a $0,30 \mathrm{ml}$ de disolución 0,05M de EDTA. Por lo tanto, este volumen de corrección debe sumarse al gastado en la valoración.

\subsection{Resultados}

Se determinó el contenido de trióxido de azufre en 10 cementos portiand, tanto por el método gravimétrico clásico como por el complexométrico propuesto. En cada muestra se hlcteron dos determinaciones por uno $\mathrm{y}$ otro procedímiento.

Los resultados se exponen en el cuadro 1.

Las diferencias entre los valores aislados obtenidos por el método gravimétrico no rebasan excepto en el caso del cemento 4 , el valor máximo de 0,10 , admitido por las normas $A . S$. $T$, $M$. (6) para dos determinaciones realizadas por un mismo operador trabajando con la misma muestra. 
Igual sucede con las diferencias entre los valores ajslados obtenidos por el método complexométrico propuesto, salvo en el caso del cemento 9.

Las dfferencias entre los valores medios hallados por el método gravimétrico $\mathrm{y}$ el complexométrico tampoco rebasan dicho valor máximo, excepto en el caso del ya mencionado cemento 4 .

Se pudo observar que este cemento contenía sulfuros, cosa no frecuente en los cementos portland, a no ser que contengan escorias granuladas de alto horno, to cual sucede en Ios cementos siderúrgicos. La presencia de sulfuros en el cemento desvirtúa los resultados de la determinación de tríóxido de azufre, por la mayor o menor oxidactón que puedan experimentar durante el proceso analítico, a no ser que se determinen por separado y se haga la corrección correspondiente. Es preciso tener esto en cuenta, particularmente en el caso de utilizar en el ataque de la muestra un medio oxidante, como lo es el ácido nítrico empleado en el procedímiento complexométrłco. En tales condiciones este método da resultados altos en comparación con el gravlmétrico, como ponen de manifiesto los valores obtenidos para el cemento 4.

\section{CUA D R O 1}

\begin{tabular}{|c|}
\hline $\begin{array}{c}\text { Cemento } \\
\text { N. }{ }^{\circ}\end{array}$ \\
\hline 1 \\
\hline 2 \\
\hline 3 \\
\hline 4 \\
\hline 5 \\
\hline 6 \\
\hline 7 \\
\hline 8 \\
\hline 9 \\
\hline 10 \\
\hline
\end{tabular}

GRAVISETIICO

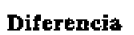

iferencia

0,02

0,06

0,04

0,22

0,10

0.00

0,02

0,08

0,06

0,00 so, $\%$

Mediax $\begin{array}{r}\text { Valores } \\ \text { aislados }\end{array}$

VOLUMETRICO

bifereneia

Medias

Diferenela

2,43

2,43

2,17

1,95

1,86

1,74

1,68

1,67

1,52

1,42

1,09
2,43

2,18

2,16

1,98

1,98

1,99

1,99

1,67

1,65

1,77
1,71

1,64

1,62

1,46
1,46

1,47

1,35

1,18

$\begin{array}{rrr}0,00 & 2,43 & 0,00 \\ 0,02 & 2,17 & 0,00 \\ 0,00 & 1,98 & +0,03 \\ 0,00 & 1,99 & +0,13 \\ 0,02 & 1,66 & -0,08 \\ 0,06 & 1,74 & +0,06 \\ 0,02 & 1,63 & -0,04 \\ 0,00 & 1,46 & -0,06 \\ 0,12 & 1,41 & -0,01 \\ 0,04 & 1,16 & +0,07\end{array}$

Esto se conflrmó de manera decisiva al llevar a cabo cinco determinaciones gravimétricas y cinco complexométricas de trióxido de azufre en otro cemento portland que contenia sulfuros. Los resultados se ponen de manifiesto en el cuadro 2 .

C U A D R O 2

\begin{tabular}{|c|c|c|c|c|c|c|c|}
\hline \multirow{2}{*}{ 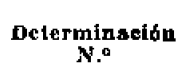 } & \multicolumn{7}{|c|}{ SO $*$} \\
\hline & \multicolumn{3}{|c|}{ Gravimétrico } & \multicolumn{3}{|c|}{ Complexométrico } & Diferencla \\
\hline 1 & 1,38 & \multirow{5}{*}{\multicolumn{2}{|c|}{ 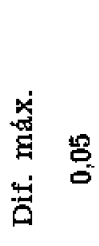 }} & 1,41 & \multirow{5}{*}{\multicolumn{2}{|c|}{ 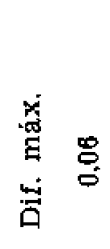 }} & $+0,03$ \\
\hline 2 & 1,36 & & & 1,45 & & & $+0,09$ \\
\hline 3 & 1,33 & & & 1,42 & & & +0.09 \\
\hline 4 & 1,33 & & & 1,47 & & & $+0,14$ \\
\hline 5 & 1,33 & & & 1,47 & & & +0.14 \\
\hline Medias & 1,34 & & & 1.44 & & & -0.10 \\
\hline
\end{tabular}


Se aprecta que las diferencias son del mismo orden que la encontrada en el caso del cemento 4 (cuadro 1) y todas positivas, es decir, que los resultados complexométricos son erróneos por exceso.

Se efectuó otra serie de cinco determinaciones, tanto gravimétricas como complexométricas, con otro cemento portland totalmente exento de sulfuros, habiéndose obtenido los resultados que se indican en el cuadro 3.

\section{CUADRO 3}

\begin{tabular}{|c|c|c|c|c|c|c|c|}
\hline \multirow{2}{*}{$\underset{\mathbf{N} \times a}{\text { Deinasión }}$} & \multicolumn{7}{|c|}{$\mathbf{s o}_{3} \%$} \\
\hline & \multicolumn{3}{|c|}{ Gravimétrien } & \multicolumn{3}{|c|}{ Complexométrico } & Diferencia \\
\hline 1 & 3,23 & \multirow{5}{*}{\multicolumn{2}{|c|}{ 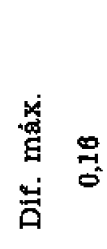 }} & 3,07 & \multirow{5}{*}{\multicolumn{2}{|c|}{ 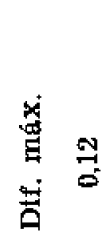 }} & $-0,16$ \\
\hline 2 & 3,13 & & & 3,19 & & & $+0,06$ \\
\hline 3 & 3,13 & & & 3,11 & & & $-0,02$ \\
\hline 4 & 3,10 & & & 3,07 & & & $-0,03$ \\
\hline 5 & 3,07 & & & 3,07 & & & 0,00 \\
\hline Medias & $\mathbf{3 , 1 3}$ & & & 3,10 & & & $-0,03$ \\
\hline
\end{tabular}

Los resultados en este caso son más semejantes y las diferencias, de signo vario, menores.

La diferencla máxima admitida por las normas $\mathbf{A}$. S. T. $\mathbf{M}$. entre valores extremos obtenidos en tres determinaclones gravimétricas realizadas por un mismo operador con una misma muestra es de $0,15 \%$. Los cuadros 2 y 3 (5 determinaciones) ponen de maniflesto lo correcto de los resultados, así como que los del método complexométrico son aceptables en pie de igualdad con los del gravimétrico, slempre que los cementos no contengan sulfuros.

Se trató también de establecer una comparación entre los resultados gravimétricos precipitando sulfato bárico y sulfato de plomo, y los complexométricos obtenidos por redisolución de este último precipitado. Se operó con dos cementos y en el caso de uno de ellos se modificaron algunas variables. Los resultados, en los que va incluída la correcclón correspondlente a la solubilldad del sulfato de plomo (33), se indican en el cuadro 4 .

\section{UA D R O 4}

\begin{tabular}{|c|c|c|c|c|c|c|}
\hline \multirow{4}{*}{$\underset{N .0^{\circ}}{\text { Cemento }}$} & \multicolumn{6}{|c|}{$\mathbf{s O}_{2} \%$} \\
\hline & \multicolumn{3}{|c|}{ GRAVIMFTRICO } & \multicolumn{3}{|c|}{ COMPLEXOMETHICO } \\
\hline & \multirow{2}{*}{ so. } & \multirow{2}{*}{$\underset{\text { so.Pb }}{\text { Il }}$} & \multirow{2}{*}{ Diferencia } & \multirow{2}{*}{$\underset{\text { Sot }}{\text { 1II }}$} & \multicolumn{2}{|c|}{ Diferencias } \\
\hline & & & & & III - I & III - II \\
\hline $1 \mathbf{a}$ & 2,43 & 2.44 & $+0,01$ & & & \\
\hline $1 b$ & 2,43 & 2,46 & $+0,02$ & 2,43 & 0,00 & $-0,03$ \\
\hline $1 \mathrm{c}$ & 2,43 & 2,36 & $-0,07$ & & & \\
\hline 2 & 2,17 & 2,02 & $-0,15$ & 2,17 & 0,00 & $+0,15$ \\
\hline
\end{tabular}

En el caso la se filtró el precipitado de sulfato de plomo a través de papel y se operó según (37), calclnando el precipitado a $650^{\circ} \mathrm{C}$ durante treinta minutos.

En los restantes casos se filtró a través de flitro de vidrio Pirex (núm. 2 en el caso $1 \mathrm{~b}$, núm. 4 en el 1c y núm. 3 en el 2) y se desecó en estufa a $100^{\circ} \mathrm{C}$ pesando el precipitado de sulfato de plomo. En 1b y 2 se disolvió después el precipitado, y con la disoluclón se procedió a la determinación complexométrica de $\mathrm{SO}_{3}$, según queda expuesto en lo que antecede.

Los tiempos de ataque de la muestra, y de sedimentación del precipitado de sulfato de plomo fueron, en los casos $1 \mathrm{a}, 1 \mathrm{~b}, 1 \mathrm{c}$ y 2 , de cuarenta minutos y una hora, media hora y cuarenta y cinco minutos, media hora y una hora, y medla hora y media hora, respectivamente.

Los resultados gravimétricos hallados por preclpitación de sulfato bárico y los volumétricos obtenldos por complexometría del sulfato de plomo disuelto son perfectamente concordantes. 


\section{Conclusiones}

La determinación volumétrica (complexométrica) de trióxido de azufre en el cemento portland por precipitación en forma de sulfato de plomo, redisolución de éste y valoración complexométriea del plomo, tiene, desde el punto de vista de los autores, ventajas sobre otros procedimientos también complexométricos, encaminados al mismo fin.

Por otra parte, el método propuesto, como volumétrico, es más sencillo y rápido que el gravimétrico clásico y está en pie de igualdad con éste en cuanto a precisión de resultados, permitiendo el ahorro de tiempo y de operaciones tales como pesadas y calcinaciones, asi como el del instrumental indispensable para las mismas. No exige preparación especial por parte de los operadores corrientes de los Laboratorios de Control de fábrica.

Por todo ello, su interés para los mismos queda claramente de manifiesto.

Es de advertir el error por exceso que puede cometerse en el caso de que los cementos en que se determina el trióxido de azufre contengan sulfuros.

bibliografía

(1) Calleja, J.: Memorias de la III Reunión Internacional sobre Reactividad de los Sólidos. Madrid, 1956.

(2) LERCH, W.: Proceedings A. S. T. M., 46, 1946.

(3) Calleja, J.: Materiales de Construcción (I. T. C. C.), núms. 76, 77 y 78, 1956.

(4) GurNeA, S.: Comunicación privada, 1955.

(5) Calleja, J.: Materiales de Construcción (I. T. C. C.), núms. 96 y 97,1959 .

(6) A. S. T. M. Standard C114-58, pág. 71, 1958.

(7) Ibíd., pág. 93.

(8) Rudy, B.: Journ. Res. Nat. Bureau Standards, $16,555-56,1936$

(9) Blondiau, L.: Rev. Mat. Const., 2-4, 1947.

(10) VOGEL, E., y Schleisen, K. J.: Silikattechnik, $8(6), 241-242,1957$.

(11) Lesar, D.: South African Ind. Chem., 11, 236245, 1957.

(12) WallRaf, M.: Zement-Kalk-Gips, núm. 2, 55$56,1959$.

(13) Sredel, K.: Zement-Kalk-Gips, núm. 4, 89, 1951 .

(14) Frey, W.: Zeitsch. Angew. Chem., 64, 203, 1952.

(15) Shaw, N.: Anal. Chem., núm. 10, 1682, 1858

(16) Donskaya, E. V., y Volkova, M. G.: Zurn. Prikl. Xim. (U. R. S. S.), 29 (10), 1598, 1957.

(17) Bogdanova, I. V.: Rev. Quim. Anal. (U. R. S. S.), $14(3), 373,1959$.

(18) Prede, R.: Schweiz. Arch., 25 (7), 221, 1959.

(19) LANGE, H.: Silikattechník, 7, 336, 1956.

(20) Yoshro, O.: Semento Gijutsu Nenpo, 6, 69-72, 1952.

(21) MatouscheK, F.: Zement-Kalk-Gips, 43 (1),
9-19, 1954; Rev. Mat. Constr., núm. 464, 137. 1954.

(22) Smidth, F. L.: Cement Lime Manuf, 23, 122 1950.

(23) BECKER, F.: Bulletin Holderbank, 20-52, 136 , 1950.

(24) Lydersen, D., y GJerns, O.: Zeitsch. Anal. Chem., 137 (3), 189, 1952.

(25) SPILlner, F., y Vorgt, V.: Zeitsch Angew. Chem., 64, 203, 1952.

(26) Calleja, J., y Fernández París, J. M.: Revísta de Ciencia Aplicada, XIII, $(69-4), 326-888$, 1959.

(27) Cluley, H. J.: Journ. Soc. Glass, Techn XLIII, (210), 30T-36T, 1959.

(28) Bogdanova, I. V.; Malamud, M. M. y NescraDIMova, N. M.: Tsement (U. R. S S.), $25(2)$ $12,1959$.

(29) KaINZNER, A.: Zement-Kalk-Gips, 10 (7), 287 1957.

(30) Rumler, F.: Zeitsch. Anal. Chem., 166 (1), 23$24,1959$.

(31) Belcher, R.; Gibbon, D., y West, T. S.: Chimie et Ind., 127, 1954

(32) Bond, R. D.: Chimie et Ind., 941, 1955.

(33) JAckson, P. J.: Chimie et Ind., 434, 1954

(34) Schawarzenbach, G.: Die Komplexometrische Titration, 3." ed., págs. 13 y 90, F. Encke Verlag, Stuttgart, 1957.

(35) Fritz, I. S. y otros: Anal. Chem, 27, 14611462,1955, y 29, 158-161, 1957.

(36) Willard, H., y Furman, H.: Análisis Quimico Cuantitativo, 2." ed., pág. 481. M. Marín Barcelona, 1948.

(37) Treadwell, F. P.: Tratado de Química Analitica. II Análisis Cuantitativo, 7. ed., página.149. M. Marín. Barcelona, 1949. 